\title{
Nonequilibrium Collective Phenomena in the Onset of Pitting Corrosion
}

\author{
A.S. Mikhailov ${ }^{\mathrm{a}}$, J.R. Scully ${ }^{\mathrm{b}}$, and J.L. Hudson ${ }^{\mathrm{c}}$ \\ ${ }^{a}$ Abteilung Physikalische Chemie, Fritz-Haber-Institut der Max-Planck-Gesellschaft, Faradayweg 4-6, \\ 14195 Berlin, Germany \\ ${ }^{b}$ Department of Materials Science and Engineering, University of Virginia, Charlottesville, VA 22904, \\ USA \\ ${ }^{\mathrm{c}}$ Department of Chemical Engineering, University of Virginia, Charlottesville, VA 22904, USA
}

\begin{abstract}
Nonequilibrium collective phenomena and effects of nonlinear pattern formation play the crucial role in the onset of pitting corrosion on stainless steels. Such materials are naturally protected by the oxide layer covering their surface. The onset of pitting corrosion involves the development of microscopic metastable pits, each persisting for about a second. As proposed in our publications and confirmed in subsequent experiments, sudden transition to active corrosion results from chain reproduction of metastable pits on the stainless steel surface leading to an autocatalytic explosion.
\end{abstract}

\section{Introduction}

In the investigations of G. Ertl and his coworkers, it has been clearly demonstrated that nonlinear nonequilibrium phenomena are of fundamental importance for understanding of the kinetics of catalytic surface reactions that provide the basis for modern chemical technology and environmental pollution prevention. As we show in this minireview, similar concepts and ideas are essential to explain sudden onset of pitting corrosion on stainless steels - a process which is responsible for a third of all chemical plant failures and is crucial for the safety of nuclear reactors and nuclear waste disposal.

Stainless steels are naturally protected by passivating oxide layers that inhibit electrochemical reaction and thus corrosion. Nevertheless, microscopic pits, accompanied by small, short transients of current associated with metal dissolution, do occur at surface defects and inclusions and can lead to significant localized pitting corrosion. Each pit produces a small spike of a few seconds duration in the electrical current, indicating an anodic reaction, and then dies out. A schematic of a metastable pitting event is shown in Fig. 1. 


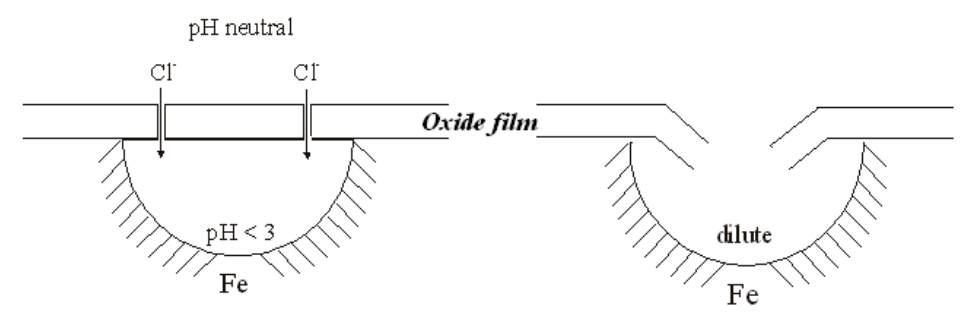

Figure 1: Schematic of the local changes that occur during a metastable pitting event.

A microscopic event begins at a surface defect in which metal is oxidized and the resultant metal cations are hydrolyzed; this produces a drop in $\mathrm{pH}$ and the flow of anions, such as $\mathrm{Cl}^{-}$, into the pit in order to maintain electro-neutrality. The acidic, high $\mathrm{Cl}^{-}$solution depassivates the protective oxide leading to rapid oxidation rates in the pits. An abrupt collapse of the protective oxide film, or other pit destabilizing event, as shown in the right portion of the figure, allows exchange of the more dilute electrolyte outside with the acidic pit electrolyte and the $\mathrm{pH}$ returns to its original, neutral condition. Nevertheless, the process does produce a small amount of aggressive species, including protons, which can diffuse from the pit and weaken adjacent oxide layers. A time trace of current during a metastable pitting event is shown in Fig. 2.

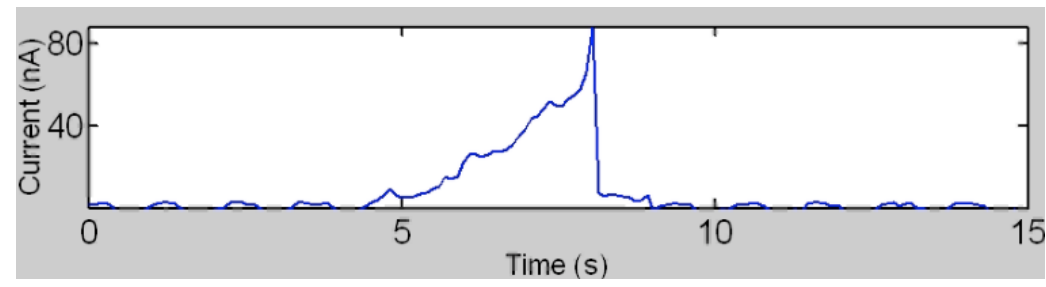

Figure 2: Current during a single (reactivated) pit on in a $0.05 \mathrm{M} \mathrm{NaCl}$ solution at $22^{\circ} \mathrm{C}$ and applied potential of $607 \mathrm{mV}_{\mathrm{NHE}}$. (1). Analogous current-time profiles for a metastable pit on AISI type 304 SS have been shown in Ref. 2.

Pitting corrosion shows a sharp rise in the number, frequency, and currents associated with these metastable events with only a small change in conditions, such as applied potential, corrodant concentrations or temperature. An example of the sudden increase in current with small change in potential is shown in Fig. 3. The experiment is carried out as a scan, that is, the potential is slowly ramped linearly upward with time. 


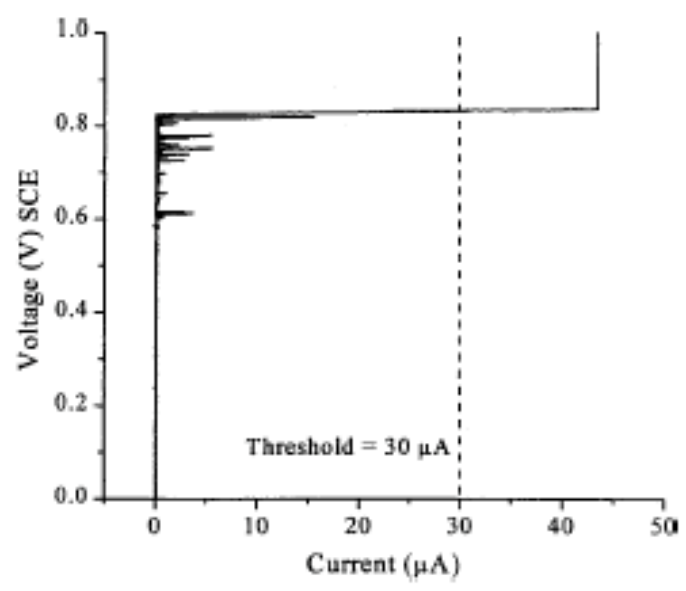

Figure 3: Potentiodynamic scan of a $0.25 \mathrm{~mm}$ diam 316 stainless steel wire dipped in a $\mathrm{pH} 6$ sodium chloride solution at $47^{\circ} \mathrm{C}$. The pitting potential is taken to be the point at which the current rises to a value greater than 30. From (3).

The pitting potential (at fixed temperature and electrolyte concentration) is an important parameter in most localized corrosion phenomena because it describes the critical transition potential, above which passive materials become severely pitted (4-7). At or near this threshold, significant changes in the surface conditions have been observed, both of which can contribute to the increase in current. Increases in pit density have been seen (8); in addition, the growth of single or small numbers of stable pits can occur $(4,9)$. In the former case, the increase in current is caused by the increase in number density; in the latter, a few pitting sites contribute larger amounts due to their increased local current. A further complication can arise, viz., stable pitting preceded by metastable pit formation, which is indicated by anodic current spikes that form micrometer size pits, often at a sub-lattice or array of non-metallic inclusions that act as weak sites in the passive film $(10,11)$. Moreover, it is well known that there is a statistical correlation between metastable and stable pitting (12) and that the frequency of metastable pitting increases with increasing applied potential (11-15). A trigger mechanism has also been proposed (16).

The important problem of the stabilization (growth) of single pits leading to an increase in total current has received considerable attention. Many authors consider stabilization to be associated with a single weakest or most favorable site that attains the conditions necessary to grow steadily (17-19). Stabilization occurs when the pit dissolution, cover, pit shape, surface roughness, and salt films all conspire to form a pit environment more aggressive than the critical chemistry needed to sustain pit growth $(2,18,20,21)$. Thus, the metastable pitting phase can lead 
to stabilization and propagation stages. Traditionally, the threshold potential in pitting has been related to pit stabilization at either a single pit or a population of pits.

The density of pits on the metal surface is known to sharply increase at the corrosion onset, suggesting that collective effects in the ensemble of metastable pitting events are essential $(18,20,21)$. The contribution of the growth of pit density is an important factor in the transition to pitting corrosion, either as a transition to high currents and/or as a precursor to transitions to stable pitting.

What processes are responsible for a sudden explosive increase in metastable pit generation rate and anodic current that occur upon slight changes in corroding conditions? In chemical kinetics and nuclear physics, such an explosive growth is usually associated with a chain reaction of autocatalytic reproduction of some particles. A decade ago, we suggested that similar autocatalytic behavior is involved in the corrosion transition: each metastable pitting event enhances the probability of appearance of other pits within a short time after it has occurred (22). Subsequently, the theory was extended to take into account that the influence of a pitting event affects only the adjacent area on the surface $(3,1)$.

Physical processes that promote or suppress cooperative growth of pit sites are explained within the acid/halide pitting mechanism (18). Aggressive species accumulation within and adjacent to active pits causes clustered formation of new pits due to a temporary presence of a more aggressive solution and subsequent persistent surface damage on nearby un-pitted surfaces. Damage of sulfide inclusions in stainless steel have been considered in many publications (2326). Surface damage in commercial stainless steels (Fe-Cr-Ni-Mo-Mn alloys) is brought about by partial dissolution of mixed metal manganese sulfide $\mathrm{Mn}(\mathrm{Cr}, \mathrm{Fe}) \mathrm{S}$ inclusions particularly in acids (15). These often form occluded sites and enable sulfide ion release that enhance pitting susceptibility and can lower $\mathrm{E}_{\text {pit }}$ determined from current rise $(25,27)$. This phenomenon can occur on other passive metals containing discrete defect sites such as precipitation age-hardened Al-alloys containing constituent particles.

In our theoretical investigations, we have predicted $(1,22)$ that the release of an aggressive species from a metastable pit and its subsequent diffusion over the surface, that causes weakening of the protective oxide layer, are already sufficient to explain sudden onset of pitting corrosion on stainless steels. These predictions have been confirmed in specially performed experiments using contrast-enhanced optical microscopy and ellipsometry for surface imaging 
(EMSI) (1,28). These ideas were further explored in order to theoretically and experimentally demonstrate the possibility of the efficient control of corrosion onset on stainless steels $(29,30)$.

In the next section, the theoretical model of metastable pitting corrosion is given and the results of its numerical simulations are presented. In Sec. 3, a short summary of optical microscopy observations, supporting the theory, is provided. The last section is devoted to the extensions of the theory to provide a new explanation for the threshold potential in pitting corrosion, which is relevant for corrosion control, and to recent related experiments that support this argument.

\section{Statistical theory of metastable pitting}

For statistical investigations of cooperative effects in a population of metastable pits on the corroding surface, one needs a simplified model that focuses only on the principal features of individual metastable pits and interactions between them $(20,21,31)$.

In this model (1), we do not differentiate between different kinds of ions which are released by active pits and assume that, when the reaction at a pit site takes place, some aggressive species is released into the solution. The local concentration $c$ of the aggressive species in the diffusion boundary layer obeys the equation:

$$
\frac{\partial c}{\partial t}=-\gamma c+D \nabla^{2} c+\frac{K}{l_{0}} \sum_{i} g\left(t-t_{i}\right) \delta\left(t-t_{i}\right)
$$

The first term on the right-hand side takes into account the loss of the aggressive species through transport into the bulk of the electrolyte; the rate constant of this process is $\gamma=2 D / l_{0}^{2}$ where $D$ is the diffusivity and $l_{0}$ is the diffusion boundary layer thickness. The second term describes lateral diffusion within the boundary layer.

The last term in equation (1) corresponds to the release of aggressive species by the pits. For simplicity, we assume that all metastable pitting events are identical in their properties. An active pit, located at point $\boldsymbol{r}_{i}$ at time $t_{i}$, releases into solution within its lifetime a total number $K$ moles of such ions. Because the pits are very small, they are pictured in the model as point sources. The function $g(t)$ defines temporal evolution of the pit activity, it is chosen in the form

$$
g(t)=\left\{\begin{array}{c}
\frac{1}{\tau_{0}} \exp \left(-t / \tau_{0}\right), t>0 \\
0, t<0
\end{array}\right.
$$

where $\tau_{0}$ is the lifetime of an active pit. 
Aggressive solution around a pit produces local weakening of the protective oxide layer in its vicinity. Introducing a phenomenological variable $s$ that specifies the degree of the local film damage, the equation for its temporal evolution is written as

$$
\frac{\partial s}{\partial t}=-\kappa s+v c .
$$

Here, $v$ is the rate constant for the oxide film damage in the presence of the aggressive species; $\kappa$ is the rate constant determining how fast the oxide film can repair itself when such aggressive species is absent.

In addition to the release and diffusion of aggressive species, the electrochemical reaction taking place in a pit lead to the Ohmic local potential drop in the solution. This potential drop is however observed only in a very narrow neighborhood of a pit and, in the simplest approximation, its effects can be neglected.

Metastable pitting events are seen in this theory as stochastic occurrences. The probability that an active pit appears within a time interval $\Delta t$ within a surface area element $\Delta S$ is given by $w \Delta S \Delta t$ where $w$ is the pit generation rate. If this rate is constant, the pits are statistically independent and they have the Poisson distribution. On the other hand, if the surface around a pit gets modified and this modification persists for a while, the generation rate can effectively keep the local memory of the previous pitting events. Then, clustering of metastable pits and, eventually, an explosion of their number become possible.

The memory effects are introduced into the model assuming that the pit generation rate depends on the local concentration $c$ of aggressive species and on the local damage $s$ of the protective oxide layer. This dependence is chosen in the form

$$
w(c, s)=\frac{w_{\max }}{1+\exp \left[\left(M_{0}-\alpha_{c} c-\alpha_{s} s\right) / H\right]} .
$$

The pitting rate increases with $s$ and $c$ and reaches saturation at $w_{\max }$. The coefficients $\alpha_{c}$ and $\alpha_{s}$ determine the sensitivity of the pitting rate to the presence of aggressive ions and the film damage. The pitting rate steeply increases when a threshold, specified by the parameter $M_{0}$. Is exceeded. The parameter $H$ sets how steep this transition is.

According to equations $(1-4)$, the probability of generation of pits is enhanced in the vicinity of an existing metastable pit. This enhancement takes place within a region with the radius given by the diffusion length of the aggressive species, i. e the distance passed by a 
released aggressive ion before it leaves the boundary layer. This distance is, by the order of magnitude, equal to the boundary layer thickness. The diffusing aggressive species is present around a pit only within a relatively short time after its release, while it is still found within the boundary layer. Therefore, the direct enhancement of pit generation by the aggressive species is only a short-term effect. The long-term memory of previous pittings arises due to the damage of the oxide layer, induced by the released aggressive species. The self-repair of the oxide layer after its damage is a slow process, with the characteristic time of about $10 \mathrm{~s}$. Within that time, the probability of creation of further pits remains significantly enhanced within the diffusion-length neighborhood of a metastable pit.

Thus, a pit favors, within the surface memory time, creation of further pits in its neighborhood. This means that the considered stochastic process is effectively autocatalytic. Chain reproduction of metastable pits, analogous to chain chemical or nuclear reactions, can therefore occur. When such reproduction prevails over the natural passivation ("death") of metastable pits, an autocatalytic explosion characterized by the exponential growth of the number of metastable pits should take place.

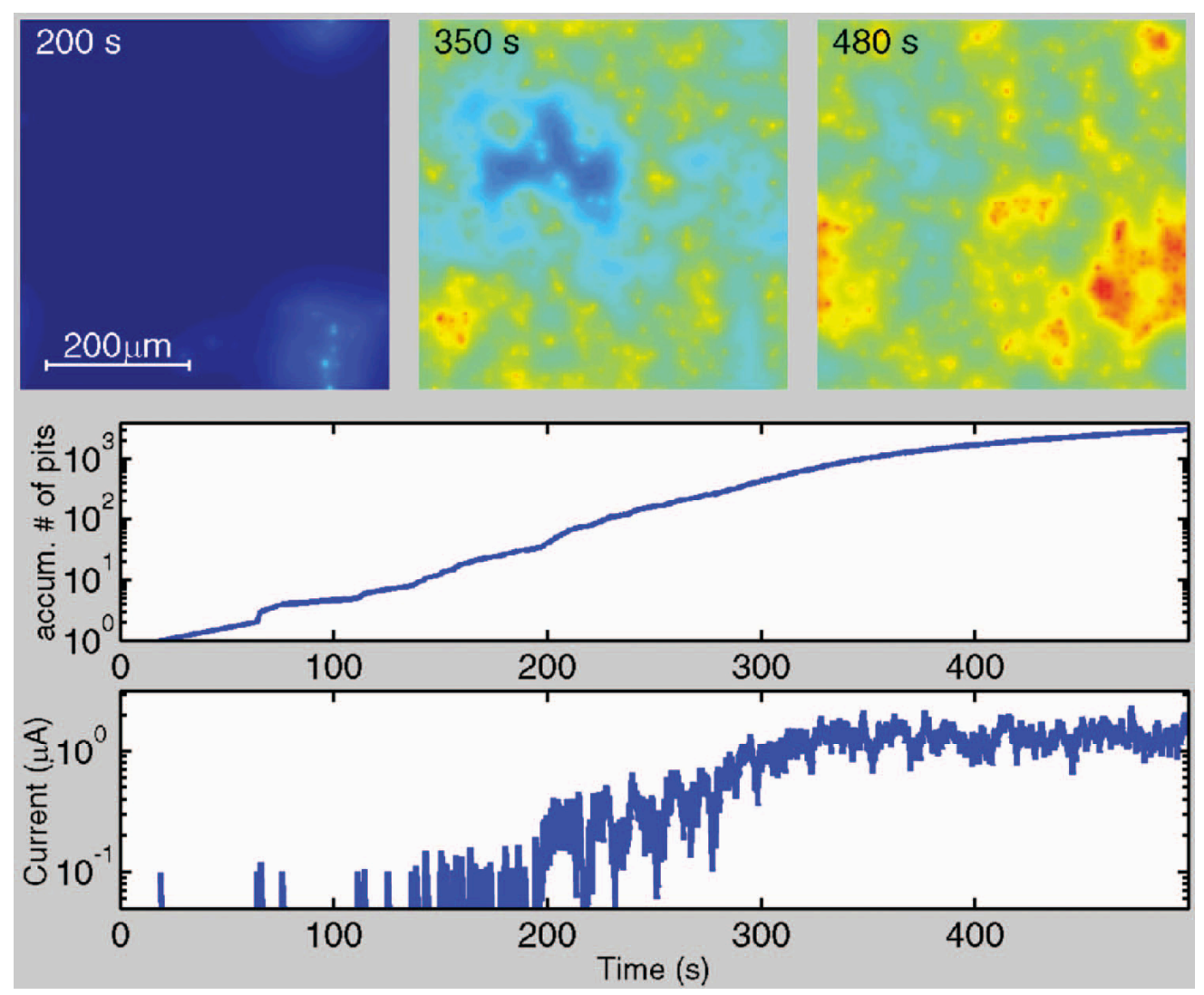


Fig. 4. Simulation of the pitting corrosion onset. Three snapshots of the oxide film damage distribution over the surface (top, red color corresponds to the higher local damage) and time dependences of the accumulated number of pits (middle) and of the current (bottom). From (1).

Figure 4 shows the data of a numerical simulation of the model where such an explosion has indeed been observed (1). The upper panels display three subsequent snapshots of the metal surface, with the local film damage visualized using a color code. The center and the bottom panels show, in the logarithmic scale, the accumulated total number of pits on the surface and the total current (that specifies the reaction intensity). Within a broad interval preceding final saturation, both plots remain linear, indicating the exponential growth.

Reproduction of metastable pits can lead to the phenomena analogous to infection spreading. If the initial perturbation (e.g., a surface scratch) is large enough, an intensive-pitting seed is created. As time goes on, the region with intensive pitting spreads away from the seed, similar to propagation of an infection front in the epidemics (Fig. 5d-f). On the other hand, a small perturbation (e.g., a short scratch) is not able to initiate corrosion spreading. Then, the initial region of intensive pittings gradually shrinks with time and finally disappears (Fig. 4a-c).

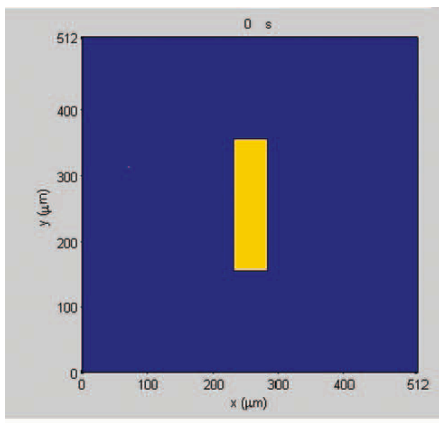

(a)

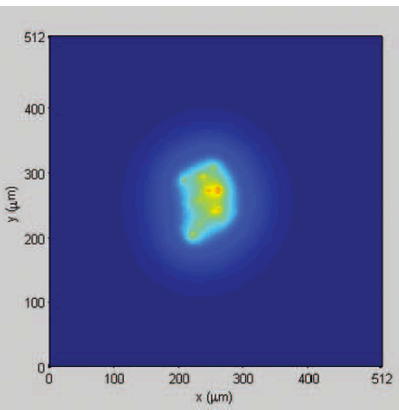

(b)

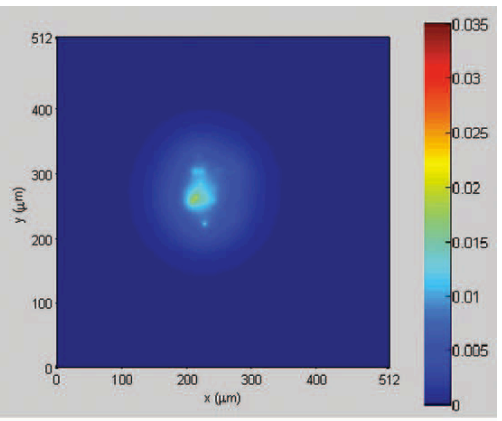

(c)

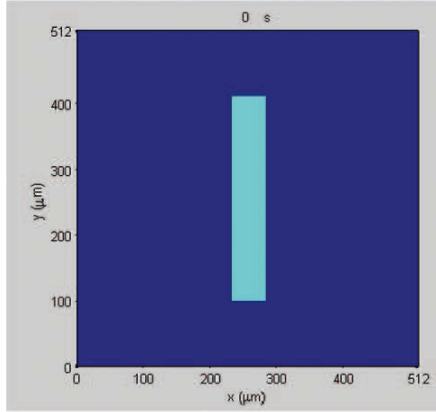

(d)

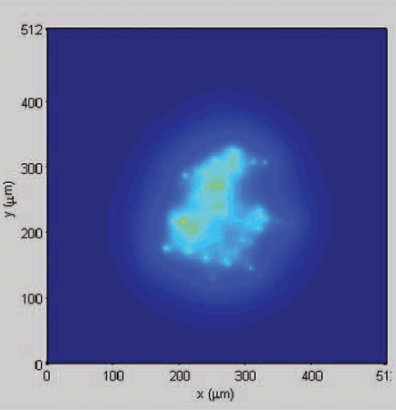

(e)

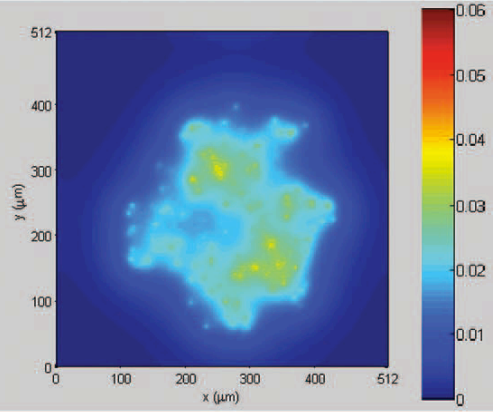

(f)

Fig. 5. Snapshots of simulations showing decay (a-c) and growth (d-f) of the metastable pitting region, starting from local damage of the protective oxide layer. From (31). 
The mean-field approximation of the considered stochastic model allows to analytically determine the speed and the profile of the spreading corrosion fronts; it also provides an analytical estimate for the critical size of the damaged region that is sufficient to initiate the corrosion spreading (31).

In the first variant of the theory which has been outlined above, we have assumed that new pits can appear anywhere on the surface. It is however known that the pits develop at local surface heterogeneities representing primarily $\mathrm{MnS}$ inclusions. The assumption that the nucleation of pits is possible anywhere on the metal surface is validated only in the limit of a high inclusion density. In commercial steels, moderate inclusion densities are typical and, therefore, the theory should be modified to take into account the surface heterogeneity.

This theory extension has been undertaken in Ref. 29 and 32. Now, pits are allowed to nucleate only at a fixed set of sites that are randomly distributed over the surface. The density of such sites and the characteristic mean distance between them represent then important additional parameters of the model.

The mean distance between the inclusions should be compared with the characteristic diffusion length of the aggressive species released in a single pitting event (this length roughly coincides with thickness of the boundary layer). If the distance between nearest inclusions is much larger than the diffusion length, aggressive ions leave the boundary layer and are convected away in the solution before they can reach the next inclusion site. Therefore, individual pitting events are independent in this case and the autocatalytic effects are absent. Therefore, a regime with low intensity of metastable pitting, such as shown in Fig. 6, is then observed in the simulations. 

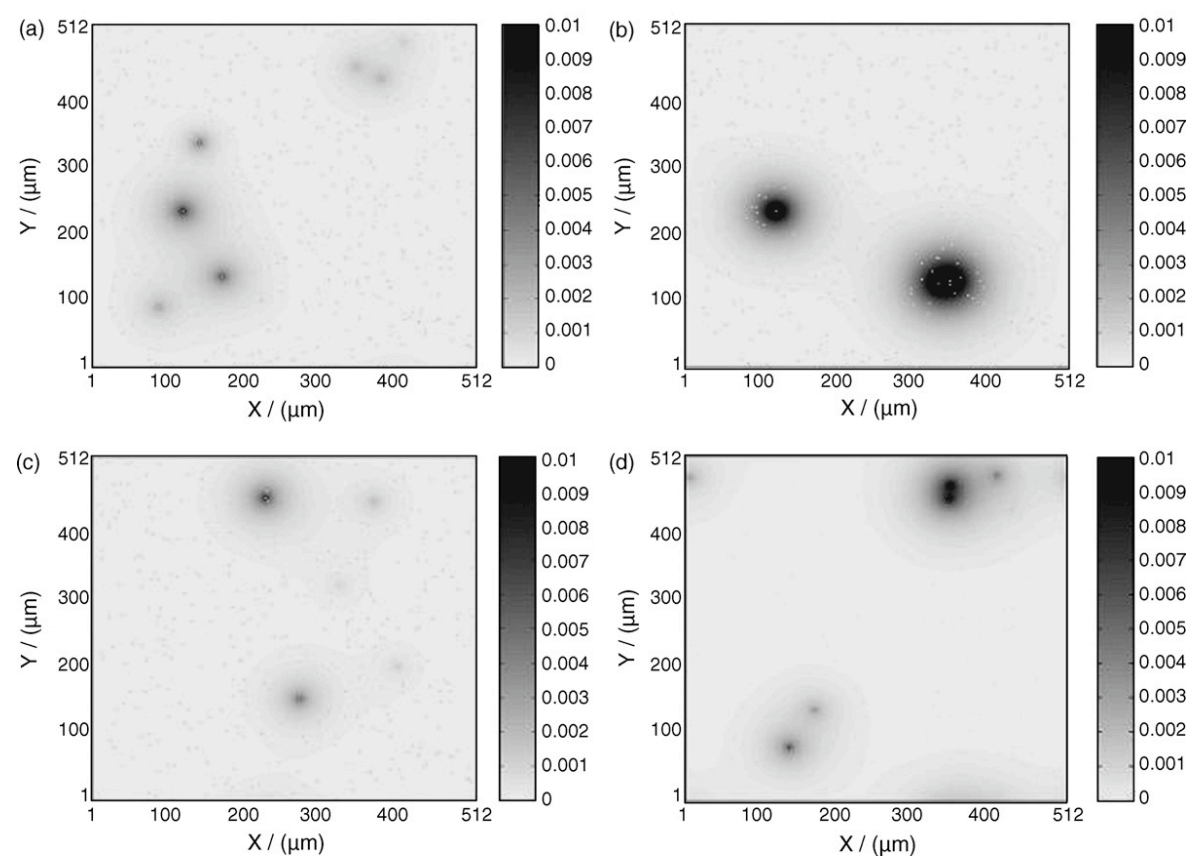

Fig. 6. Snapshots of film damage at subsequent times (a) $t=400 \mathrm{~s}$, (b) $t=800 \mathrm{~s}$, (c) $t=1200$ $\mathrm{s}$ and (d) $t=1501 \mathrm{~s}$. Inclusion density just below the critical value. From (29).

The situation is different when inclusion density is so high that the mean distance between the inclusions is shorter than the diffusion length of the aggressive species. In this case, interactions between metastable pits are strong and the autocatalytic explosion of metastable pits, leading to intensive pitting, is observed. An example of a simulation of such surface explosion is shown in Fig.7.
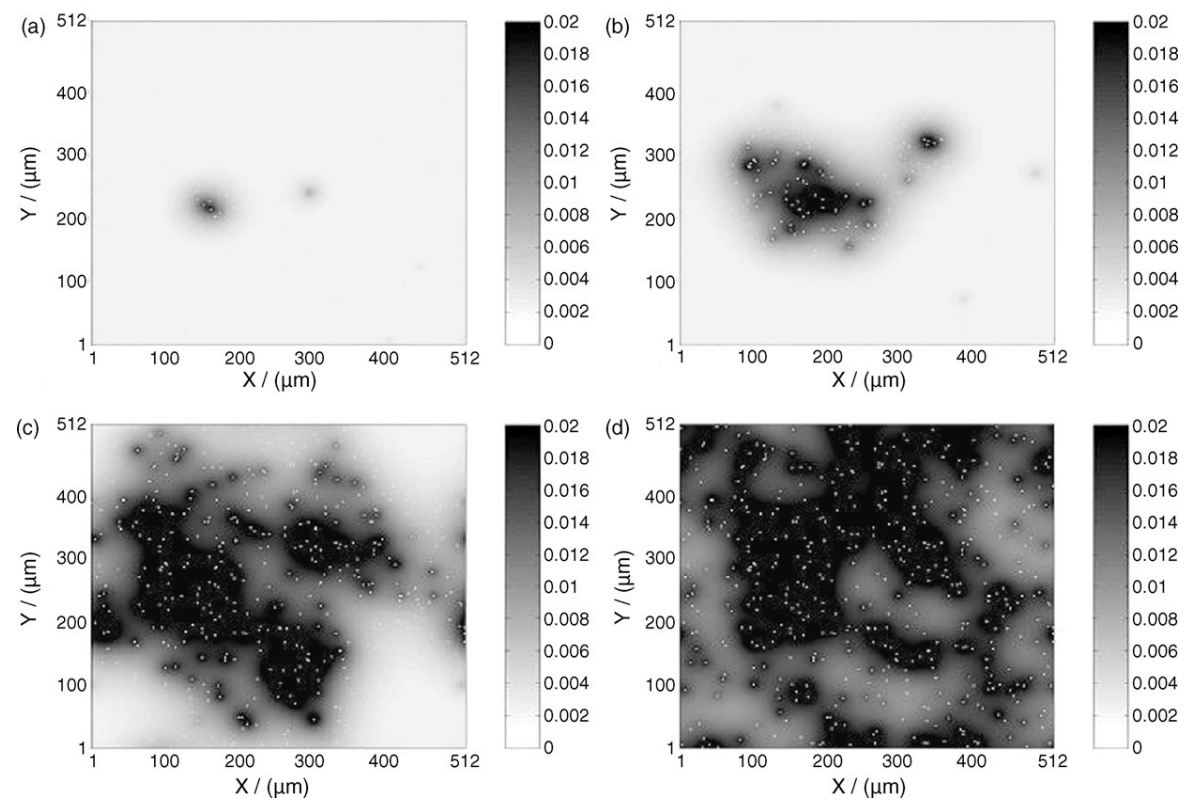
Fig. 7. Snapshots of film damage at subsequent times (a) $t=400 \mathrm{~s}$, (b) $t=600 \mathrm{~s}$, (c) $t=800 \mathrm{~s}$ and (d) $t=1200 \mathrm{~s}$. Inclusion density just above the critical value. Inclusion sites are shown as white dots. From (29).

The corrosion intensity can be characterized by the mean current per inclusion. Figure 8 shows how this mean current depends on the inclusion density in numerical simulations. A sudden strong increase in the corrosion intensity takes place when the critical inclusion density is exceeded.

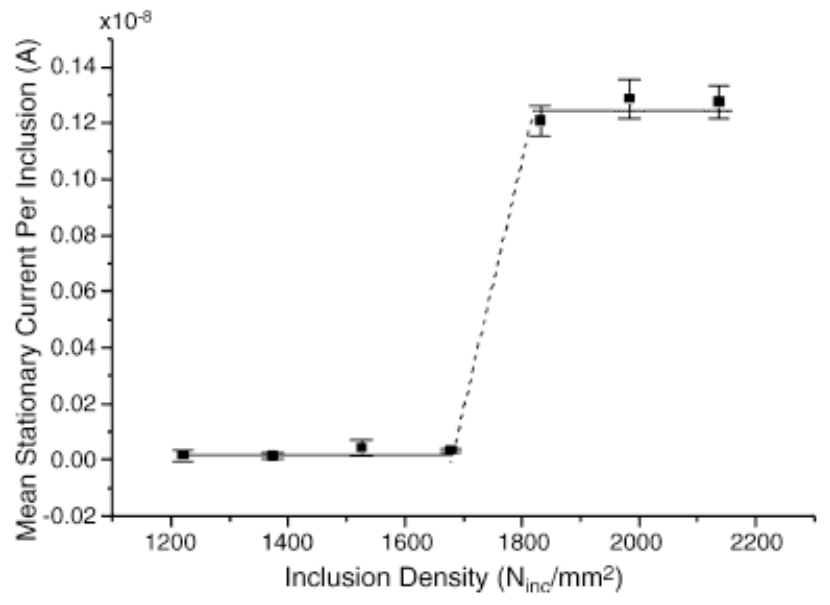

Fig. 8. Mean stationary current per inclusion as function of the inclusion density. Error bars show standard deviation for 10 simulations. From (29).

Instead of changing the inclusion density, one can vary the thickness of boundary layer while keeping the inclusion density fixed (the boundary layer can be made thinner by increasing solution stirring, see the experiments below). Figure 9 shows that a sharp increase in the corrosion intensity occurs when some critical boundary layer thickness, depending on the inclusion density, is exceeded. 


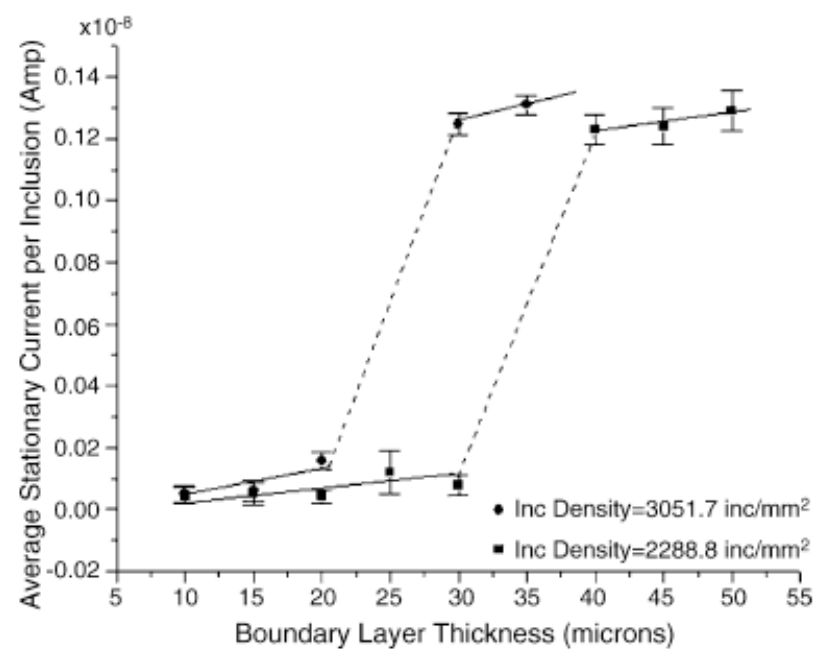

Fig. 9. Mean stationary current per inclusion as function of the boundary layer thickness for two inclusion densities. From (29).

In the model, the pits remain metastable at any pitting intensity. In reality, high intensity of metastable pitting would however result in a transition to the irreversible pitting corrosion characterized by persistent growing pits. Such a transition can still be discussed within the developed theory if we set a certain level of metastable corrosion intensity and say that physically it would correspond to the onset of irreversible corrosion. Once this is done, the dependence of the corrosion threshold on the system parameters can be numerically investigated.

Figure 10 shows how the pitting corrosion threshold (corresponding in the experiments to the critical electric potential) depends on the inclusion density. The threshold increases strongly as the inclusion density is lowered. This rapid decrease begins roughly at the inclusion density for which the mean distance between the inclusions is equal to the thickness of the boundary layer (shown by the vertical dot line in the figure). 


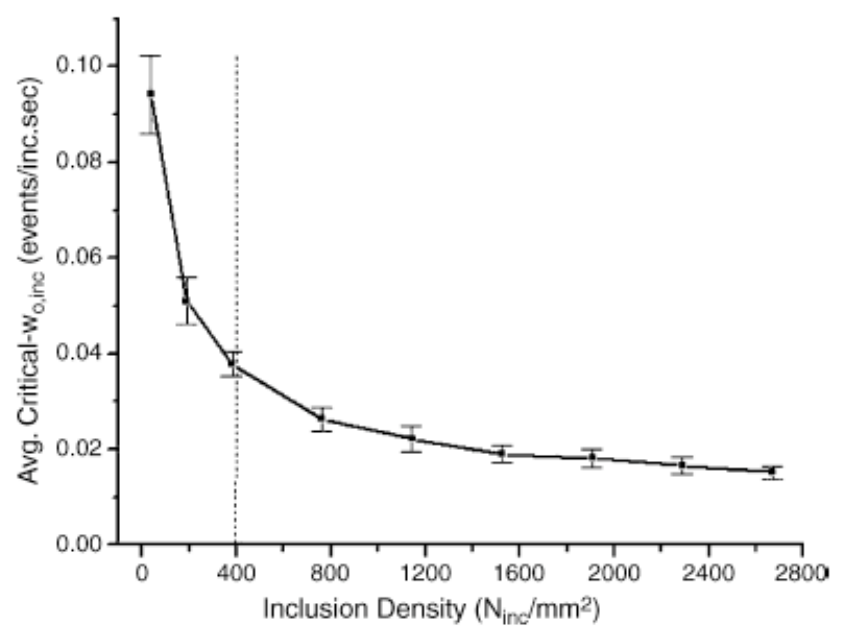

Fig. 10. Mean critical corrosion threshold as function of the inclusion density. From (29).

\section{Microscopic in situ observations of metastable pitting}

To verify theoretical predictions, microscopic in situ observations of the onset of pitting corrosion were needed. Characterization of the corrosion onset is a problem of high practical importance and it has been experimentally investigated by many groups. Characterization of the surface and observation of pitting sites have been carried out using various scanning probe methods (33-37).

The experimental group of H. H. Rotermund, which has previously performed extensive in situ microscopic observations of surface chemical reactions in the Fritz Haber Institute, performed the experiments related to the concept described above. They specially adapted the high-resolution contrastenhanced optical microscopy for direct monitoring surface processes in the electrolyte. Moreover, they used ellipsomicroscopy for surface imaging (EMSI), which permits real-time observation of ultrathin layers on surfaces, to visualize the local damage of the protective oxide layer during the corrosion onset. Both techniques were accompanied by parallel monitoring of the electric current (1).

The microscopy methods have allowed to see in real time how new pits were appearing on the surface. By recording the current generated by a single pit, it was possible to determine the interval of time within which a metastable pit remains active. After its passivation, a pit was still seen as a small black dot in the optical microscopy images. Subsequently, the same pit could undergo re-activation, generating another current pulse. In optical microscopy images, such re-activated pits were seen as surrounded by a bright halo, as long as the reaction within the pit goes on. Thus, by computer processing of the observation data, not only positions of individual pits could be determined, but also the activity state of a pit could be identified. 


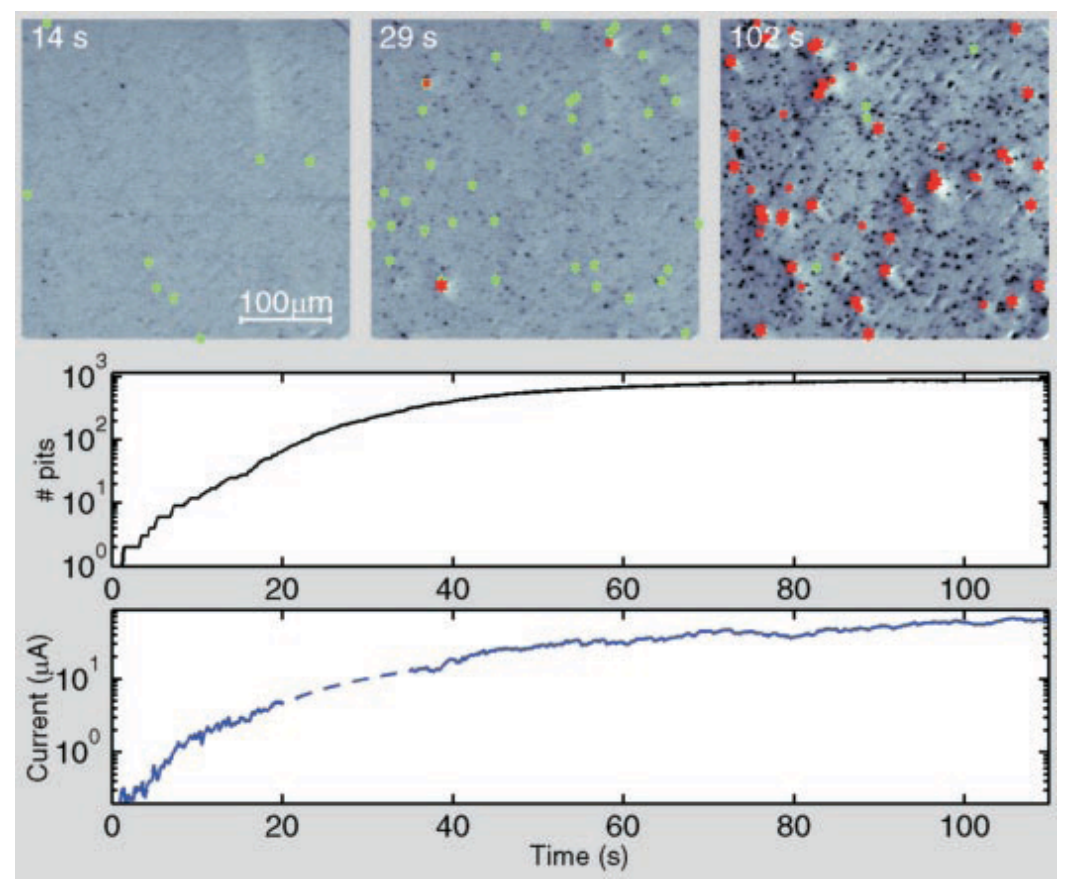

Fig. 11. Sudden onset of pitting corrosion observed with contrast-enhanced optical microscopy. From (1).

Figure 11 shows typical experimental data. The top row presents three subsequent computerprocessed optical microscopy images. Green stars show the newly created pits, red stars indicate the locations of reactivated pits. The two plots display, in the logarithmic scale, the temporal dependences of the total number of pits and of the total current. The initial exponential increase in the number of pits and the reaction rate are clearly seen here. Comparing Figs. 4 and 11, a good agreement with the theoretical predictions can be noticed. Thus, the sudden onset of pitting corrosion is indeed associated with an exponential increase in the number of metastable pits.

Processing the data, lifetimes of metastable pits (i.e., intervals of time within a pit remains active) could be determined. This analysis has revealed that, as the number of metastable pits rapidly increases, their lifetimes do not significantly change. Hence, the previous theoretical view, according to which the onset of local corrosion and the associated increase in current are due to the stabilization of metastable pits, is not applicable to our experiments.

The spatial resolution of EMSI is limited to about $12 \mathrm{~m}$ and, therefore, individual pits could not be resolved by using this method. Continuously monitoring the metal surface with EMSI, it could however noticed that diffuse bright area, indicating local surface modification, developed on the surface and their appearance was correlated with an increase of the electric current (1). 


\section{(a)}
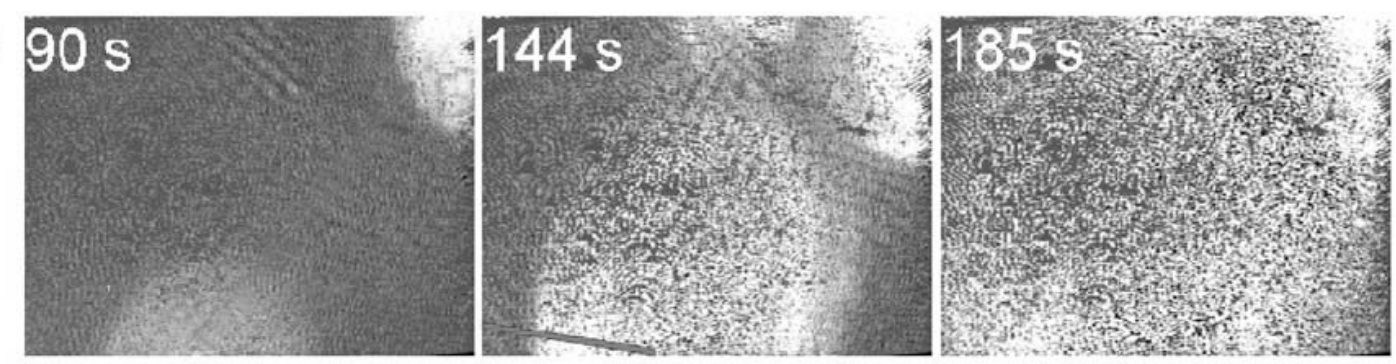

(b)
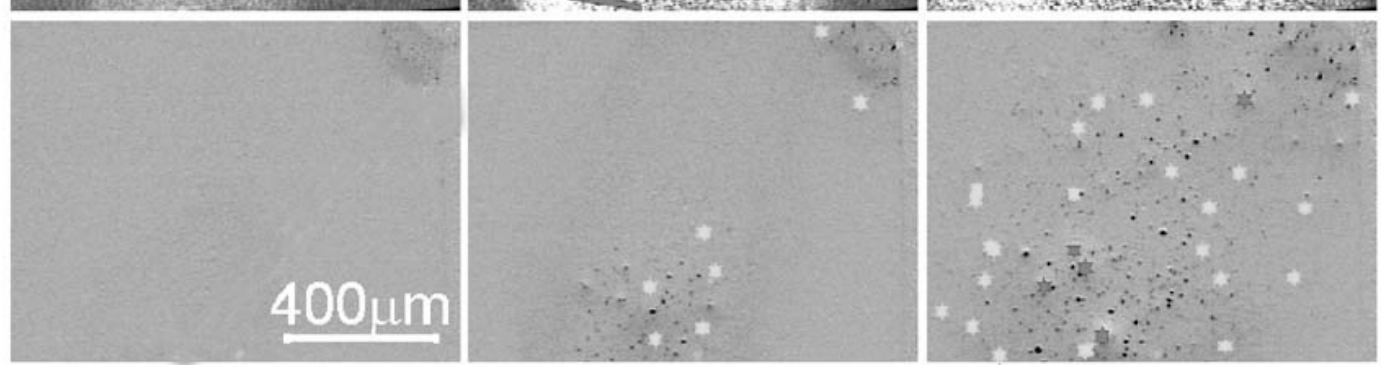

Fig. 12. Onset of pitting corrosion observed simultaneously with EMSI and optical microscopy. White stars in the optical microscopy images (below) indicate newly created pits, gray stars show reactivated pits. From (28), with minor changes.

Subsequently, the experimental set-up has been modified, allowing simultaneous real-time observation of the surface with EMSI and the optical microscopy (28). Figure 12 shows an example of such an observation. Spreading fronts, leaving behind a modified surface, are clearly seen in the EMSI images (top row). The parallel optical microscopy images (bottom row) provide direct evidence that pit generation occurs mostly within such modified regions and that region covered with pits spreads together with the propagating fronts.

\section{Experimental control of pitting corrosion onset}

In this section we discuss how the abrupt onset of pitting corrosion depends on the relationship of the effective nearest neighbor distance of inclusions to the characteristic lateral diffusion length of reaction products. The former depends on the metallurgical properties of the steel and specific surface finishing steps taken; the latter can be controlled by changing the rate of mass transfer between electrolyte and reaction surface. 


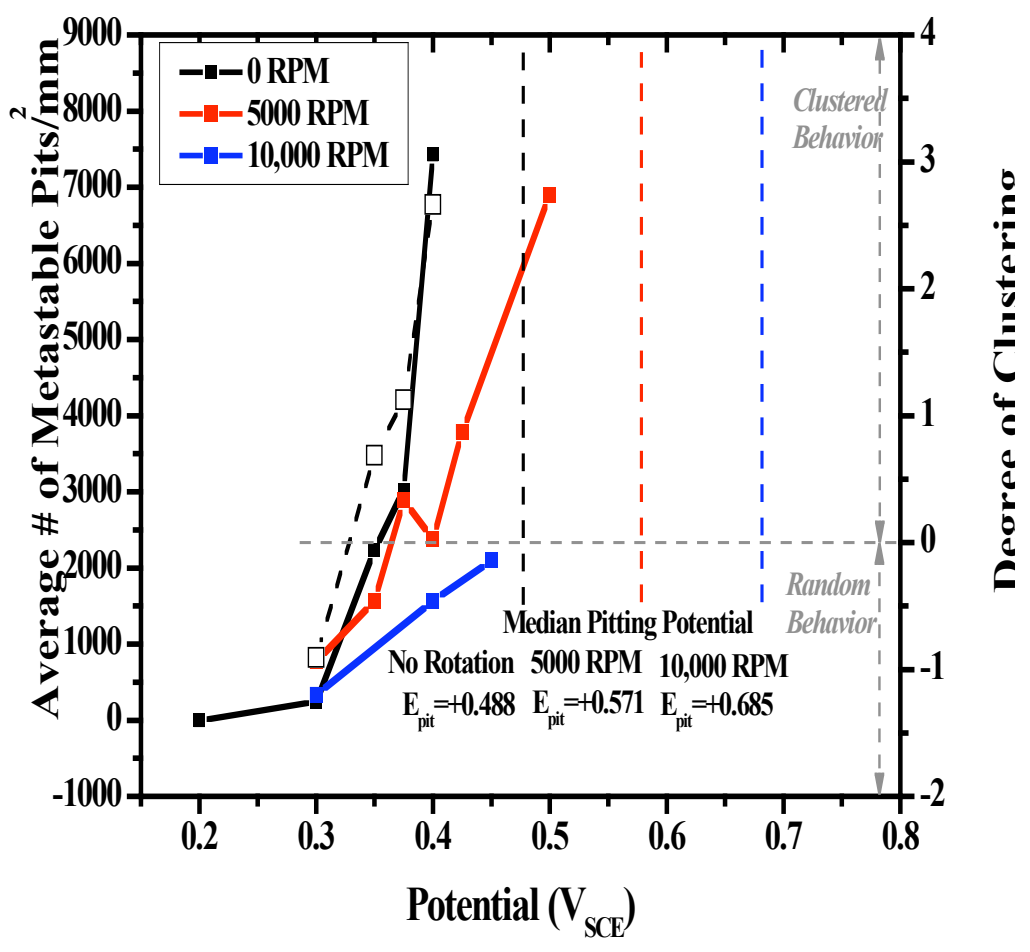

Figure 13. Pitting of commercial 316 stainless steel, $250 \mu \mathrm{m}$ wire. $(0.13 \% \mathrm{C}, 0.31 \% \mathrm{Co}, 18.18 \%$ $\mathrm{Cr}, 0.38 \% \mathrm{Cu}, 1.75 \% \mathrm{Mn}, 2.40 \% \mathrm{Mo}, 12.25 \% \mathrm{Ni}, 0.016 \% \mathrm{~S}, 0.35 \% \mathrm{Si}$, balance Fe in wt.\%). The effective nearest neighbor distance of inclusions (mean distance between an inclusion and its five closest neighbors) is $\mathrm{NND}=17.3 \mu \mathrm{m} .0 .05 \mathrm{M} \mathrm{NaCl}(\mathrm{pH} 6.8)$ at $47^{\circ} \mathrm{C}$ Scans carried out after potentiostatic holds for 3600 seconds at rotation rates and potentials indicated. The average number of metastable pits $/ \mathrm{mm}^{2}$ (closed symbols) after one hour exposure at the rotation rates indicated and degree of pit site clustering (open squares) under 0 rotation rate at various constant potentials. From (30).

Figures 13 and 14 demonstrate the collective behavior of metastable pitting on 316 stainless steel wire (30). Such stainless steel alloys have a mixture of ( $\mathrm{Mn}, \mathrm{Fe}, \mathrm{Cr}) \mathrm{S}$, silicon rich and oxide inclusions that form an array of defect sites (38). MnS inclusions are the primary pit initiation sites in stainless steels and can make up anywhere from $20 \%$ to $100 \%$ of the inclusions (14). The $\mathrm{MnS}$ inclusion density of the commercial 316 stainless steel alloy was $2800 \mathrm{inclusions} / \mathrm{mm}^{2}$ with an average "effective" nearest neighbor distance (NND) of $17.3 \mu \mathrm{m}$ taken as the average distance between an inclusion and its five closest neighbors. Other model alloys studied had inclusion nearest neighbor distances ranging from near infinity for a $\mathrm{MnS}$ inclusion free alloy to 8.0-27.2 $\mu \mathrm{m}$ at low, intermediate, and high sulfur contents. These experiments were done with a rotating disk electrode configuration; the boundary layer thickness ( $\delta$ ), and thus the lateral spreading of reaction products within this boundary layer, depends on the rotation rate; increased rotation rate 
decreases the mass transfer boundary layer thickness and thus the extent of lateral spreading of reaction products $(30,39)$.
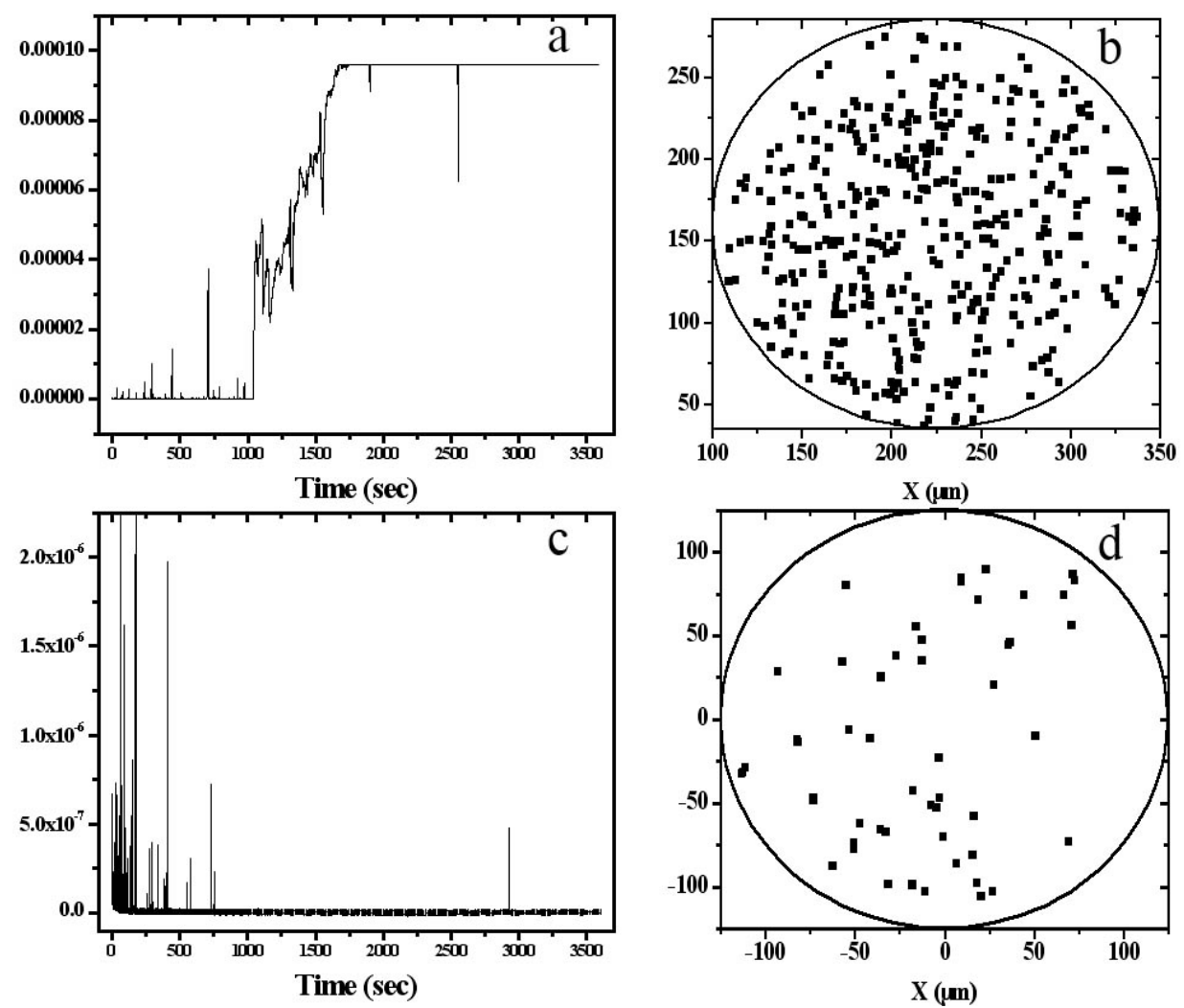

Figure 14. (a,c) Current-time behavior; each spike indicates a pit formation event. location of pit sites (b, d) after one hour exposure at constant potential conducted on commercial 316 stainless steel (inclusion nearest neighbor distance $\mathrm{NND}=17.3 \mu \mathrm{m}$ ). Pit clustering is indicated in (b) but not (d) as indicated by statistical analyses (a-b) exposure conducted at $+0.4 \mathrm{~V}_{\mathrm{SCE}}$ in $0.05 \mathrm{M} \mathrm{NaCl}$ at $47^{\circ} \mathrm{C}$ without rotation $(\delta=125$ $\mu \mathrm{m})$. (c-d) exposure conducted at $+0.4 \mathrm{~V}_{\mathrm{SCE}}$ in $0.05 \mathrm{M} \mathrm{NaCl}$ on a rotating disk at $5000 \mathrm{RPM}(\delta=8.9 \mu \mathrm{m})$. From (30).

The number density of metastable pits after the given time period as a function of applied potential during a scan is shown in Figure $13 \mathrm{a}$ at rotation rates $(0,5,000$, and 10,000 RPM).

Median values of $\mathrm{E}_{\mathrm{pit}}$ (taken as the potential associated with the current rise) for the commercial 316 stainless steel $(\mathrm{NND}=17.3 \mu \mathrm{m})$ in $\mathrm{NaCl}$ solution increased with rotation rate from +0.488 to 0.571 , and, finally, to $0.685 \mathrm{~V}_{\mathrm{SCE}}$ (volts vs. saturated calomel reference electrode) corresponding to no rotation $(\delta=125 \mu \mathrm{m}), 5000 \mathrm{RPM}(\delta=8.9 \mu \mathrm{m})$, and 10,000 RPM $(\delta=6.3$ $\mu \mathrm{m}$ ), respectively (Figure 13). A sudden growth in the number of metastable pit sites $/ \mathrm{mm}^{2}$ was seen with increasing potential as the applied potential approached the $\mathrm{E}_{\mathrm{pit}}$ (closed symbols in Figures 13). (See also $(1,8)$.) Moreover, the distribution of pit sites changed from spatially random to clustered as $\mathrm{E}_{\mathrm{pit}}$ was approached (Figure 13 (open symbols)). However, $\mathrm{E}_{\text {pit}}$, 
associated with the rapid current rise, the critical transition potential associated with explosive growth in metastable pit sites (Figure 1a), as well as the critical potential at which pit sites became clustered were all shifted to more positive potentials upon stirring, i.e., as the diffusion boundary layer thickness, $\delta$, decreased.

Results of potentiostatic tests on commercial stainless steel under both rotating and stationary conditions that reinforce these findings are shown in Figure 14. At a potential of $+0.3 \mathrm{~V}_{\mathrm{SCE}}$, far below the critical transition potential, very few pit sites initiate under both stagnant and rotating conditions. Pit sites are randomly distributed (40). At slightly higher potentials (e.g., +0.375 $\mathrm{V}_{\mathrm{SCE}}$ ), electrodes exhibit many more pits, and more pit interactions and pit clustering occur. Applied potentials $\left(+0.4 \mathrm{~V}_{\mathrm{SCE}}\right)$ that begin to approach the critical potential and $\mathrm{E}_{\text {pit }}$ produce bursts in current and strong clustering of pits under stagnant conditions as shown in Figures 14ab. A rapid rise in the current is observed after a period of time and continuous metastable pit spikes are observed indicating strong cooperative interactions between pit sites (Figure 14a). In contrast, the degree of cooperative interactions is reduced and the current-time series showed fewer and fewer clustered metastable pit current spikes when the solution was stirred at 5000 RPM $(\delta=8.9 \mu \mathrm{m})$ as shown in Fig.14c-d. The effective lateral diffusion length was reduced from $125 \mu \mathrm{m}$ at no rotation to $8.9 \mu \mathrm{m}$ at $5000 \mathrm{RPM}$ stirring rate. As the rotation rate was increased the diffusion boundary layer thickness was decreased until it was smaller than the defect nearest neighbor distance of $17.3 \mu \mathrm{m}$ in this stainless steel. The transition to pitting corrosion was suppressed.

A related trend is found when a series of alloys with different inclusion nearest neighbor distances are studied at a fixed rotation rate. The effect of inclusion density is shown in Figure 15. $\mathrm{E}_{\mathrm{pit}}$ increases abruptly as the inclusion density is reduced such that the inclusion nearest neighbor distance becomes larger than the characteristic lateral diffusion length, taken to be the mass transfer boundary layer thickness. These results substantiate the finding that $\mathrm{E}_{\mathrm{pit}}$ increases significantly when the characteristic diffusion length is reduced below the inclusion nearest neighbor distance. 


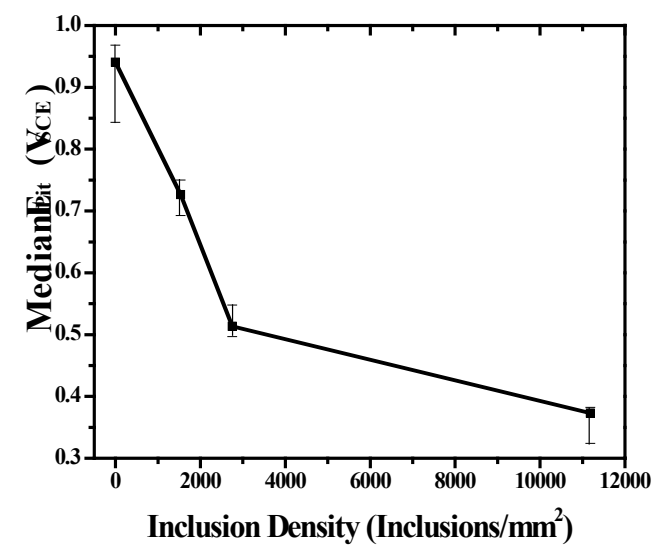

Figure 15. Median experimental pitting potential vs. inclusion density for model stainless steel alloys in $0.05 \mathrm{M} \mathrm{NaCl}$ solution at room temperature and fixed rotation rate of $1000 \mathrm{RPM}$ (diffusion length $19.8 \mu \mathrm{m}$ ). $\mathrm{E}_{\text {pit }}$, potential associated with explosive growth of pits, and pit clustering are indicate by the square symbols. $\mathrm{E}_{\mathrm{pit}}$ increases significantly when the NND increases from $16.7 \mu \mathrm{m}\left(3000 \mathrm{~mm}^{-2}\right)$ to $>20 \mu \mathrm{m}\left(1600 \mathrm{~mm}^{-2}\right)$ in otherwise identical alloys. Error bars indicate the $25 \%$ and $75 \%$ cumulative probability limits where explosive pit growth was observed experimentally in replicate experiments. From (30).

\section{Discussion and Conclusions}

As our analysis has shown, full understanding of a highly important chemical process corrosion of stainless steel - cannot be achieved without relying on general concepts of nonequilibrium nonlinear pattern formation. The sudden onset of pitting corrosion observed in our experiments is not explained in terms of the properties and the behavior of single metastable pits. This abrupt transition is not due to the stabilization of individual pits and their persistent growth. Interactions between the pits and the surface memory are essential here. Such behavior is expected to occur in many commonly used engineering materials. Release of aggressive ions from an active pit and their diffusion within its neighborhood lead to the local damage of the protective oxide layer. This damage persists much longer than the time within which the released aggressive ions are locally present in the solution. Within this relatively long memory time, the surface remains highly susceptible for pitting and new pits are more easily created. Thus, some form of autocatalytic behavior is characteristic for populations of metastable pits on the stainless steel surface: each active pit increases the probability of creation of further pits within the surface memory time in its vicinity. Thus, an effective chain reaction of pits reproduction may set in the system. When chain reproduction prevails over spontaneous passivation of metastable 
pits, their autocatalytic explosion can occur on the metal surface. It is this explosion that determines the sudden onset of pitting corrosion of the steel. At the onset, the pits remain metastable and only their number gets strongly increased, giving rise to a dramatic increase in the corrosion reaction rate.

With the new explanation for the onset of pitting corrosion, problems of corrosion suppression and design of corrosion-resistant steels acquire a different view. It has been known for a long time that pits predominantly appear at the inclusions (particularly, MnS) on the metal surface. Therefore, one could expect that reducing the number of such inclusions would make the steel more corrosion resistant. Traditionally, corrosion onset was seen as associated with the independent behavior at single inclusions. Therefore, the material could be made corrosionresistant, according to this view, only by eliminating such inclusions completely or making their density as low as possible. In our approach, the corrosion onset is determined by "communication" between the inclusions through the release and diffusion of aggressive ions. Such communication extends however only over a finite region around a pit, with the radius roughly equal to the diffusion length of aggressive ions within the laminar boundary layer near the metal surface. If the mean distance between the inclusions on the surface is longer than the diffusion length, interactions between them are not efficient and autocatalytic explosions cannot develop. This implies that there is a critical density of $\mathrm{MnS}$ inclusions. The threshold of corrosion onset is strongly raised once the inclusion density is reduced below such critical value. The experiments have fully confirmed this theoretical prediction (cf. Figs. 15 and 10).

Furthermore, the diffusion length of aggressive species within the laminar boundary layer can be easily controlled. Indeed, it just coincides with the thickness of this layer. When mechanical stirring of the electrolyte is intensified, this makes the boundary layer thinner and, therefore, also shortens the diffusion length defining the "communication distance" between individual inclusions. The theory predicts that the corrosion threshold gets sharply increased when this length gets shorter than the mean distance between the inclusions, if the surface density of the latter is fixed. This is indeed what has been seen in our experiments.

Thus, to control the corrosion onset and to suppress it, one can efficiently use processes affecting interactions between metastable pits, in addition to considering properties of individual pits. 
Our investigations reveal that already a very common physical object - commercially available stainless steels - gives rise to rich nonequilibrium pattern formation phenomena on electrochemically active surface. Persistent front propagations, resembling spreading of epidemic waves and analogous to the waves demonstrated for the $\mathrm{CO}$ oxidation on $\mathrm{Pt}$ and other catalytic surface reactions, take place in such active surfaces. It would be interesting to extend the analysis to the corrosion processes on other metals and alloys by following the already developed theoretical concepts

\section{Acknowledgements}

We thank the United States Department of Energy, Office of Basic Energy Sciences, Division of Materials Sciences and Engineering for partial support under contract DEFG02-00ER45825 with Dr. Jane G. Zhu as contact monitor. We thank Swati Jain for her help in manuscript preparation.

\section{References}

[1] C. Punckt, M. Bolscher, H.H. Rotermund, A.S. Mikhailov, L. Organ, N.D. Budiansky, J.R. Scully, J.L. Hudson, Science 305 (2004) 1133-1136.

[2] P.C. Pistorius, G.T. Burnstein, Philisophical Transactions: Physical Sciences and Engineering 341 (1992) 531-559.

[3] T.T. Lunt, J.R. Scully, V. Brusamarello, A.S. Mikhailov, J.L. Hudson, Journal of Electrochemical Society 149 (2002) B163-B173.

[4] Z. Szklarska-Smilalowska, Pitting Corrosion of Metals, Houston, Texas, NACE, 1986.

[5] A. Broli, H. Holtan, Corrosion Science 17 (1977) 59-69.

[6] R.J. Brigham, E.W. Tozer, Corrosion 29 (1973) 33-36.

[7] G.K. Glass, N.R. Buenfeld, Corrosion Science 39 (1997) 1001-1013.

[8] G. Herbsleb, W. Schewenk, STAHL EISEN 87 (1967) 709-713.

[9] N.J. Laycock, R.C. Newman, Localized Corrosion of Austenitic Stainless Steels, NACE, 2001, 165-190.

[10] G.S. Eklund, Journal of Electrochemical Society 121 (1974) 467-473.

[11] G.S. Frankel, Journal of Electrochemical Society 145 (1998) 2186-2198.

[12] D.E. Williams, J. Stewart, P.H. Balkwill, Corrosion Science 36 (1994) 1213-1235.

[13] P.H. Balkwill, C. Westcott, D.E. Williams, Stocastic Approach to the Initition of Pitting Corrosion, Oxforshire, OX11 ORA, United Kingdom Atomic Energy Authority Hartwell, 1988.

[14] J. Stewart, D.E. Williams, Corrosion Science 33 (1992) 457-474.

[15] B. Baroux, Corrosion Science 28 (1988) 969-986.

[16] D.E. Williams, T.F. Mohiuddin, Y.Y. Zhu, Journal of Electrochemical Society 145 (1998) 2664-2672. 
[17] H. Boehni, F. Hunkeler, Growth Kinetics and Stability of Localized Corrosion Processes, Orlando Florida, NACE, 1990, 69-76.

[18] J.R. Galvele, Journal of Electrochemical Society 123 (1976) 464-474.

[19] G.T. Gaudet, W.T. Mo, T.A. Hatton, J.W. Tester, J. Tilly, H.S. Isaacs, R.C. Newman, American Institute of Chemical Engineers Journal 32 (1986) 949-958.

[20] N.J. Laycock, S.P. White, J.S. Noh, P.T. Wilson, R.C. Newman, Journal of Electrochemical Society 145 (1998) 1101-1108.

[21] P. Ernst, N.J. Laycock, M.H. Moayed, R.C. Newman, Corrosion Science 39 (1997) 11131136.

[22] B. Wu, J.R. Scully, J.L. Hudson, A.S. Mikhailov, Journal of Electrochemical Society 144 (1997) 1614-1620.

[23] B. Baroux, in: P. Marcus, J. Oudar (Eds.), Corrosion Mechanisms in Theory and Practice, New York, 1995, pp. 265-309.

[24] C.S. Brossia, Effects of MnS Inclusions on Crevic Corrosion of Stainless Steels in Neutral Chloride Solutions, The Electrochemical Society, 1998, 327-338.

[25] E.G. Webb, R.C. Alkire, Journal of Electrochemical Society 149 (2002) B272-B279.

[26] E.G. Webb, C.H. Paik, R.C. Alkire, Electrochemical and Solid State Letters 4 (2001) B15-B18.

[27] P. Marcus, A. Teissier, J. Oudar, Corrosion Science 24 (1984) 259-268.

[28] M. Dornhege, C. Punckt, J.L. Hudson, H.H. Rotermund, Journal of Electrochemical Society 154 (2007) C24-C27.

[29] L. Organ, Y. Tiwary, J.R. Scully, A.S. Mikhailov, J.L. Hudson, Electrochimica Acta 52 (2007) 6784-6792.

[30] J.R. Scully, N.D. Budiansky, Y. Tiwary, A.S. Mikhailov, J.L. Hudson, Corrosion Science 50 (2008) 316-324.

[31] A.S. Mikhailov, S. Jain, L. Organ, J.L. Hudson, Chaos 16 (2006) 037104.

[32] Y. Tiwary, Metastable Pitting of Heterogeneous Surfaces: Effect of Inclusions, M.S. Thesis, Charlottesville, VA, University of Virginia (2005).

[33] I. Serebrennikova, H.S. White, Electrochemical and Solid State Letters 4 (2001) B4-B6.

[34] G.S. Chen, M. Gao, R.P. Wei., Corrosion 52 (1996) 8-15.

[35] S.B. Basame, H.S. White, Langmuir 15 (1999) 819-825.

[36] N. Casillas, S.J. Charlebois, W.H. Smyrl, H.S. White, Journal of Electrochemical Society 140 (1993) L142-L145.

[37] N. Casillas, S.J. Charlebois, W.H. Smyrl, H.S. White, Journal of Electrochemical Society 141 (1994) 636-642.

[38] A.J. Sedriks, Corrosion of Stainless Steels, Princeton, NJ, A Wiley-Interscience Publication John Wiley \& Sons, Inc., 1996.

[39] E. Gileadi, Electrode Kinetics for Chemists, Chemical Engineers, and Materials Scientists, New York, VCH, 1993. 
[40] N.D. Budiansky, L. Organ, J.L. Hudson, J.R. Scully, Journal of Electrochemical Society 152 (2005) B152-B160. 